\title{
Acute post operative unilateral submandibular gland swelling following acoustic schwannoma excision under general anesthesia
}

\begin{abstract}
Acute unilateral neck swelling and sialadenitis are rare but documented causes in the differential diagnosis of acute neck swelling following surgeries performed in various surgical positions and also in critical care units. Although rare, they can lead to severe morbidity as well as mortality if not promptly addressed.

We report a case of acute unilateral submandibular gland swelling detected in the immediate post operative period following Acoustic Schwannoma excision performed via retromastoid suboccipital craniotomy in park bench position, and its subsequent management.
\end{abstract}

Keywords: Cerebellopontine Angle mass, Acoustic Schwannoma, Retrosigmoid approach, Park bench position Unilateral Submandibular gland swelling

\author{
Volume 5 Issue 3 - 2016
}

\author{
Dhruba Lahkar,' Rishab Kedia² \\ 'Department of Neuroanaesthesia and Critical Care, Medanta \\ the Medicity, India \\ ${ }^{2}$ Department of Neurosurgery, Medanta the Medicity, India
}

\begin{abstract}
Correspondence: Dhruba Lahkar, Associate Consultant Department of Neuroanaesthesia and Critical Care, Medanta the Medicity, Sec 38 Gurgaon, Haryana, India, Tel +9l |244|4|4|4; +9| 989|4|3877, Fax 9| |24 4834| ||, Email drdhruv@hotmail.com
\end{abstract}

Received: August 0I, 2016 | Published: November 10, 2016

\section{Introduction}

Acute unilateral neck swelling and sialadenitis are rare but documented causes in the differential diagnosis of acute neck swelling following surgeries performed in various surgical positions and also in critical care units. Although rare, they can lead to severe morbidity as well as mortality if not promptly addressed.

We report a case of acute unilateral submandibular gland swelling detected in the immediate post operative period following Acoustic Schwannoma excision performed via retromastoid suboccipital craniotomy with patient in park bench position and its subsequent management.

\section{Case presentation}

A 38 year old woman, with Diabetes Mellitus II, presented to our neurosurgical department with complaints of right sided hearing loss for 12 years with exacerbation from last 4 months, headache for 4-5 years especially in the right hemicranium, and unsteady gait with a tendency to fall to right side. She was operated for CSOM in 1992. There was no history of memory disturbance, visual loss, speech difficulty, swallowing difficulty, altered sensorium or seizures. MRI brain revealed a Right Cerebellopontine angle mass, suggestive of Acoustic Schwannoma.

Right Cerebellopontine angle mass excision was planned under general anaesthesia. Pre anesthetic checkup was done. CNS examination revealed Right Cranial Nerve VIII deficiency with decreased hearing and absent corneal reflex. She also exhibited right disdiadochokinesia and unsteady gait with a tendency to fall to right. All laboratory and radiological parameters performed as per institutional protocol were found to be normal.

After obtaining all the necessary informed consents, she underwent craniotomy and near total excision of the Right cerebropontine angle mass by retrosigmoid suboccipital approach under general anaesthesia. Surgery was performed in left 3/4 Park-Bench position, with the head secured with Mayfield three-pin skull clamp at a slight face-down angle, a $15^{\circ}$ lateral flexion and a $20^{\circ}$ rotation of the neck to the opposite side.
Surgery and anaesthesia were uneventful and she was extubated in the operation theatre. Thereafter, she was shifted to the neurointensive care unit for post operative management. Subsequently, within an hour of shifting from the operation theatre, patient complained of pain on the left side of the face. Examination of face showed a swelling on the left side. External examination of the swelling did not reveal any hemorrhagic spots or crepitus .Oropharyngeal examination did not reveal any edema or inflammation. There was no evidence of any stridorous breathing.

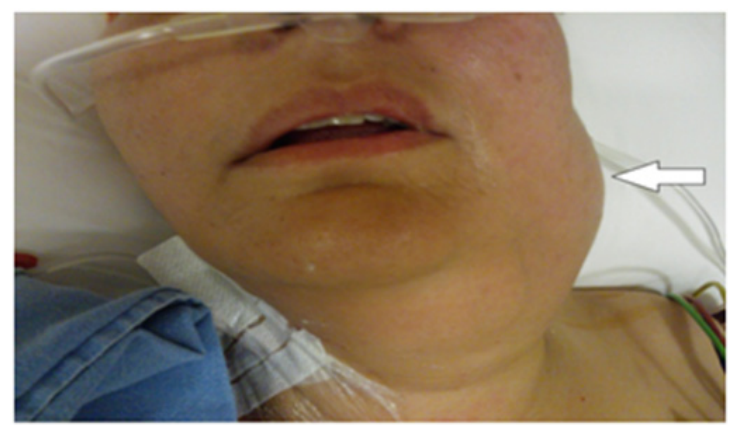

Figure I Acute Left side Neck swelling indicated by arrow.

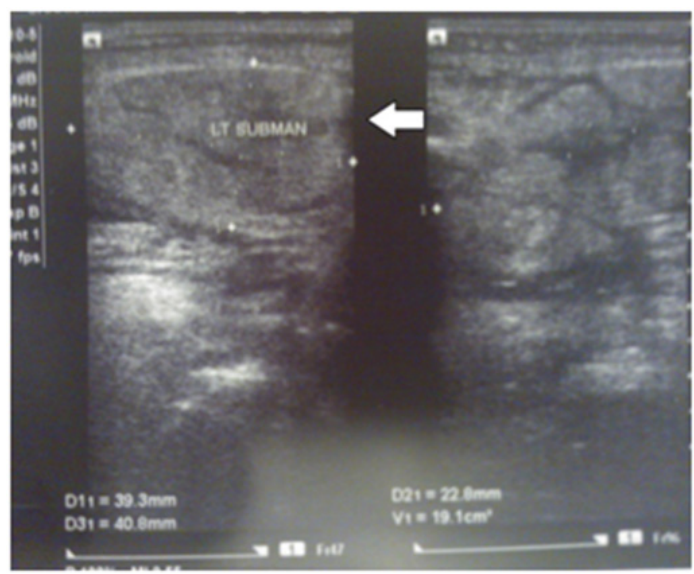

Figure 2 USG showing enlarged left submandibular gland indicated by arrow. 


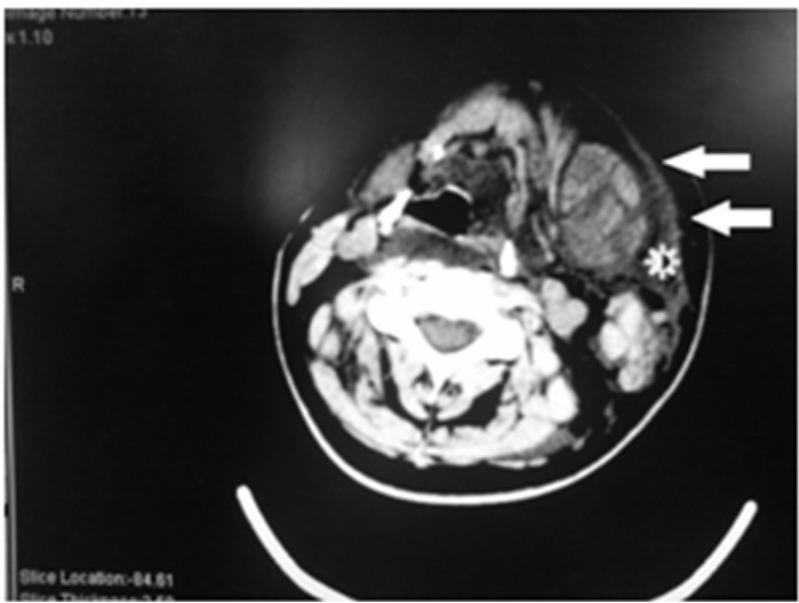

Figure 3 CT showing heterogeneously enhancing enlarged left submandibular gland (indicated by arrow) with fascial cellulitis and phlegmatous collection involving cervical spaces on left side, extending to right side and prevertebral space (Danger Space).

\section{Discussion}

Acute swelling of the face, neck and tongue are reported entities in patients undergoing various neurosurgical procedures in prone, lateral and park bench positions. It has also been reported in various non neurological procedures as well as in intensive care settings. ${ }^{1-8}$

There are five major etiologic categories of neck swelling: 9 (1) Angioedema (2) Vascular lesion (3) infection (4) denervation and (5) lymphedema. Kim et al. ${ }^{1}$ has postulated that contralateral submandibular and neck swelling after retromastoid and far-lateral approaches to the posterior fossa, where the neck is rotated and flexed, is most probably due to sialadenitis. They attributed this to obstruction of Wharton's duct due to extreme rotation of the head and compression by endotracheal tube on the tongue, which cause stasis of secretions leading to secondary bacterial infection. However in our case, USG didn't reveal any ductal obstruction although subsequent CT did reveal fascial cellulitis and phlegmatous collection in the left submandibular gland with mild parotid gland swelling.

Further intraoperatively, general anesthesia was conducted using reinforced Flexometallic tube for prevention of inadvertent kinking. Post operative oral and radiological examination also did not reveal any macroglossia. Raad et al. ${ }^{10}$ has postulated many numerous medical conditions associated with the predisposition to salivary stasis and thus an increased risk of sialadenitis: diabetes mellitus, hepatic failure, renal failure, hypothyroidism, Sjogren's syndrome, depression, and malnutrition. Perioperative use of medications with anticholinergic and diuretics effects can cause systemic dehydration, which increases the risk of salivary stasis.

Our patient had not received any anticholinergic and diuretics in her perioperative phase. The only known risk factor present was Diabetes Mellitus which was also under reasonably under control as evidenced by a preoperative HbA1c 6.2. Further our patient was 39 years old and was not obese, which rules out decreased salivary secretions and an increased risk of sialadenitis due to old age or morbid obesity. ${ }^{11}$ Our case was diagnosed on the basis of clinical and radiological findings and no identifiable cause could be found. She recovered with conservative management without any overt infective signs and symptoms to suggest infective sialadenitis.
Mei-Ching Huang et al. ${ }^{9}$ reported a case of a 42 -year-old patient who underwent a 6.5 hour surgery for excision of right acoustic neuroma in left-side down lateral position. She required reintubation within 3 hours post extubation due to development of acute left unilateral neck swelling and stridor. She remained under ventilatory support for 14 days .In absence of any precipitating factors; they proposed angioedema to be a possible cause for this acute neck swelling. They further theorized that long period of compression of the tongue and related focal soft tissue by oral endotracheal tube and extreme forward flexion of the neck during surgery can lead to just venous and/or lymphatic stasis with collapsed small veins and lymphatic ducts without any venous thrombosis. This changes the capillary permeability and results in excessive amounts of fluid escaping from the intravascular circulation. The fluid gets accumulated in the intercellular spaces, causing vasogenic edema leading to facial and neck swelling.

Although our patient responded to conservative management without any respiratory complications, the CT findings in our case lends support to this theory proposed by Huang et al. ${ }^{9}$ Retrosigmoid and far lateral cranial procedures necessitates extreme head rotations to achieve the required surgical position. Unfotunately, this can lead to rare but potentially devastating complications like acute unilateral hemifacial swelling. Surgeries like cerebellopontine angle tumors excision has inherent risks of long surgical duration and lower cranial nerve paresis or palsies in the post operative period. This, sometimes manifest as requirement of post operative ventilatory support or even reintubation due to airway compromise. Acute neck swelling and sialadenitis are like adding insult to injury in such cases, leading to significant morbidity, as evidenced by case reports and communications in various journals.

Maintenance of adequate hydration, care to avoid surgical position related soft tissue compression and endotracheal tube kinking and maintenance of neck vessel patency in the perioperative period is of paramount importance. In the postoperative period, awareness of this rare but potentially life threatening complication, maintenance of strict vigil with regards to respiratory and airway compromise, adequate hydration and adequate antibiotic prophylaxis can go a long way in reducing fatal outcomes. Our report is an attempt to highlight this condition although the course of such swelling is generally fortunately benign.

\section{Acknowledgments}

None.

\section{Conflicts of interest}

None.

\section{References}

1. Kim LJ, Klopfenstein JD, Feiz-Erfan I, Postoperative acute sialadenitis after skull base surgery. Skull Base. 2008;18(2):129-34.

2. Cavaliere F, Conti G, Annetta MG, et al. Massive facial edema and airway obstruction secondary to acute postoperative sialadenitis or "anesthesia mumps": a case report. J Med Case Rep. 2009;3:70-73.

3. Prabhu K, Ramamani, Nair S, Chacko AG. Acute submandibular sialadenitis as a cause of unilateral neck swelling after posterior fossa surgery in sitting position. Neurology India. 2010;58(6):963-964.

4. Postaci A, Aytac I, Oztekin CV, et al. Acute unilateral parotid gland swelling after lateral decubitus position under general anesthesia. Saudi J Anaesth. 2012;6(3):295-297. 
5. Serin S, Kaya S, Kara CO, et al. A Case of Anesthesia Mumps. Anesth Analg. 2007;104(4):1005.

6. Vinu Jamwal, Vijant Singh Chandail. Transient Swelling of Parotid Gland after Upper GI Endoscopy -“Anaesthesia Mumps": Time to Stay Cool. Journal of Digestive Endoscopy. 2012;3(2):30-32.

7. Ghatak T, Gurjar M, Samanta S, et al. A case of "anesthesia mumps" from ICU. Saudi J Anaesth. 2013;7(2):222-223.

8. Koramutla Pradeep Kumar, P Kishore Kumar, Gudaru Jagadesh. Acute sialadenitis of parotid gland: Anaesthesia mumps. Indian Journal of Anaesthesia. 2014;58(1):97-98.
9. Mei-Ching Huang, Yung-Cheng Wang, Jing-Shan Huang, et al. A Rare Presentation of Hemifacial Swelling Following Neurosurgery in Lateral Position: A Case Report. Clinical Molecular Medicine. 2012;3(1):7-9.

10. Raad II, Sabbagh MF, Caranasos GJ. Acute bacterial sialadenitis: a study of 29 cases and review. Rev Infect Dis. 1990;12(4):591-601.

11. Lundgren A, Kylen P, Odkvist LM. Nosocomial parotitis. Acta Otolaryngol. 1976;82(3-4):275-278. 